\title{
The Gravity Separation Mixture Fluid: An Innovative Method and Device to Separate the Components in a Gas, Liquid or Vapour Mixture
}

\author{
Stefano Farné \\ Department of Industrial, Electrical, Computer and Biomedical Engineering, University of Pavia, Italy. \\ DOI: http://doi.org/10.38177/AJBSR.2021.3404
}

Copyright: () 2021 Stefano Farné. This is an open access article distributed under the terms of the Creative Commons Attribution License, which permits unrestricted use, distribution, and reproduction in any medium, provided the original author and source are credited.

\section{ABSTRACT}

The Gravity Separation Mixture Fluid (GSMF) system, patented by Stefano Farné and Vito Lavanga, is a device that allows to separate a mixture into its different components with different specific weight, exploiting the stationing of the fluid in the spaces created by each of the two packs of honeycomb present which, in addition to increasing the surface useful for the separation of the mixture, provides the vertical space useful for eliminating the horizontal motions that would make the gravimetric separation process vain. The flow necessary for the passage inside the device is guaranteed by inlet and outlet draining pipes from which to extract the different phases of the mixture, arranged in an arrangement for three-dimensional reverse return. The process described is fundamental in many areas of industry, from agri-food, to the purification of urban wastewater or petroleum processes.

Keywords: Separator, Fluid separation, Gravimetric separator, Mixture.

\section{The Separation Process}

The need to separate fluid mixtures is widespread in industry and in several of its processes. It leads to the separation of mixtures into single substances, or of homogeneous sets of components of raw materials, based on their physical or chemical-physical characteristics.

The separation operations are opposed to the natural tendency of substances to mix intimately and spontaneously with each other which is a typical manifestation of the second law of thermodynamics. Their relevance is also visible in the ecological sector since they are applied in the removal of pollutants contained in a liquid or gaseous stream.

The operations of filtration, centrifugation, decantation, flotation, liquid-liquid or liquid-solid solvent extraction and distillation belong to this group of operating techniques.

- Membrane filtration: It is the unitary operation by which water and some solutes in a solution are selectively removed through very fine semipermeable membranes (cross-flow filtration). The main methods for concentrating with membranes are ultrafiltration and reverse osmosis.

- Centrifugation: It is the separation of heterogeneous mixtures (liquid-liquid, liquid-solid) by applying a centrifugal force. The physical principle used is based on the acceleration of gravity through the rapid rotation of a rotating drum.

- Decanting: It is a unitary operation based on the separation of a solid from a liquid or of two liquids that cannot be mixed together thanks to the force of gravity (sedimentation or surfacing).

- Flotation: It is an operation that is carried out by blowing air into a container containing a solid or colloidal mixture in suspension, the components with greater aerophilicity are dragged towards the surface of the tank 
(formation of foam), while those with greater wettability (hydrophilic) precipitate to the bottom (formation of "turbidity").

- Liquid-liquid solvent extraction: It is a unitary operation that allows separating two liquids that are miscible from each other using a solvent that preferably dissolves one of the two. The extraction of a liquid substance from a liquid mixture is called "liquid phase extraction". In the food industry it is applied to refine raw seed oils or to extract aromas from vegetables.

- Distillation: It is a system of separation of a liquid from a mixture of liquids, exploiting their different boiling temperatures. Distillation separates two liquids more easily the more different their boiling points are. Currently the distillation plants, or columns, have reached considerable dimensions which can be observed for example in refineries where fuels are produced that feed the strong energy need of our society.

Of these processes, the centrifugation and decantation / sedimentation process use a difference in density or specific gravity, as in the case of GSMF.

\section{Gravimetric Separators}

Gravimetric separation is one of the most popular separation techniques. In fact, it exploits the use of gravity, everywhere present on Earth without the aid of external actions, which makes the process extremely economical. However, it can generally require high retention times of the mixture inside the devices to make the separation effective.

The equipment that carries out phase separation by exploiting the sedimentation capacity of the mixture due to the difference in specific weight differ, first of all, in

- Continuous separators: in which the exit of the different phases, and therefore the entry of the mixture to be separated, does not cause a machine stop, or in any case forms a continuous flow and does not require control valves for the release of the different separate phases (Fig.1);



Fig.1. Continuous Gravimetric Separator

- Discontinuous separators (separating funnel): in which the introduction of the mixture to be separated and the discharge of the different phases does not occur continuously but periodically, for example after having allowed the 
necessary sedimentation time to pass for the mixture to separate in a decanter, and it is allowed and controlled by valves applied to the outlet pipes (Fig.2).

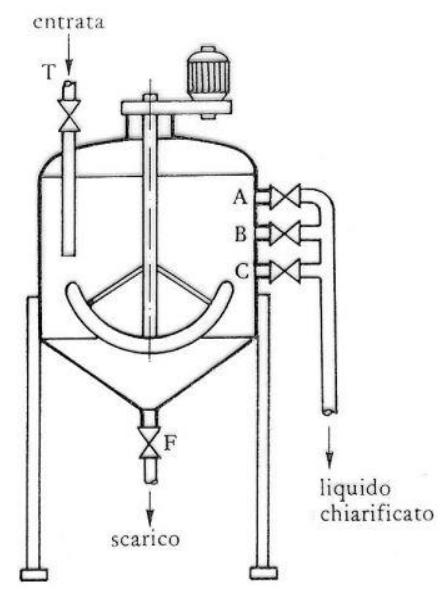

Fig.2. Discontinuous Gravimetric Separator

A further differentiation is carried out by considering the number of phases that they are able to isolate, in fact there are separators for two-phase or three-phase mixtures.

- The two-phase separators are used for example to eliminate quantities of gases trapped in the hydrocarbon flow during petroleum processes, to separate two immiscible fluid substances, such as oily substances emulsified with aqueous substances or to separate two gases having different specific weights, such as carbon dioxide and methane produced by the digestion of biomass.

Based on the difference in specific weight existing between the two phases to be separated, there are vertical, horizontal or spherical two-phase mix separators.

Vertical separators are used in processes where the ratio of gas to liquid is low or to remove sand or solid sediments within the mixture. They have a low separation efficiency, especially compared to the horizontal ones, are expensive to build and difficult to install due to their weight. The spherical ones have a spherical tank, and are used in small systems and at moderate pressures. They are difficult to design precisely and offer space limitations that affect efficiency. Furthermore, they are the most expensive to build, they have the lowest achievable efficiency even if they are the easiest to install due to their small size. Horizontal gravimetric separators, on the other hand, are the most used, especially when the ratio between the phases is high, due to the larger surface available for gravimetric separation. They also differ from the vertical ones in the direction of separation of the phases, which for a horizontal separator are perpendicular to the flow and not parallel to it as in vertical devices, which usually leads to a greater purity of the extracted phases compared to the vertical ones. In them the flow to be separated follows a horizontal path during which it separates, and elements such as disks, flat perforated pipes, curved tubes or others, the different phases reach different parts of the device to be extracted through ducts controlled by valves.

- The three-phase mix separators divide the mixes into three phases. They too can be vertical, horizontal or spherical, but they have the best efficiency and constructive choice if made for a horizontal flow. An example in 
which a three-phase separation is required can always be in the petrochemical industry for the separation of refining products such as water, oil and gas. The operation between two-phase and three-phase devices is almost identical, in fact many times a two-phase separator is used in a three-phase one to facilitate the separation of two of the three phases (such as droplets of liquid, smaller than that separable from the three-phase separator, left in the gas phase) and improve the efficiency of the device.

\section{Three-Phase Gas-Oil-Water Gravimetric Separator}

In the technical literature, it can be found how the design of gravimetric separators, while exploiting relatively simple and natural physical principles, is articulated above all due to the various factors to be taken into consideration during the design and choice of the construction criteria of these devices to make the process work and efficient.

Furthermore, the gravimetric separation, despite being a subject much analyzed and studied by various experts and engineers, presents a strong uncertainty of the results that are greatly affected by the surrounding conditions and the fluid to be separated, such as minimal variations in the entry speed of the flow or its temperature. With the recent developments of fluid dynamics simulation (CFD) software this phenomenon is becoming more and more easily understood and above all different case studies can be analyzed, with different data and device configurations from time to time, to then understand which is the most convenient design choice.

In fig. 3 there is an analysis of a three-phase gas-oil/oil/hydrocarbon-water gravimetric separator which is an excellent example to understand how its operation can easily be affected by the different design choices of these devices (Ahmed, Makwashi e M.Hameed, 2017).

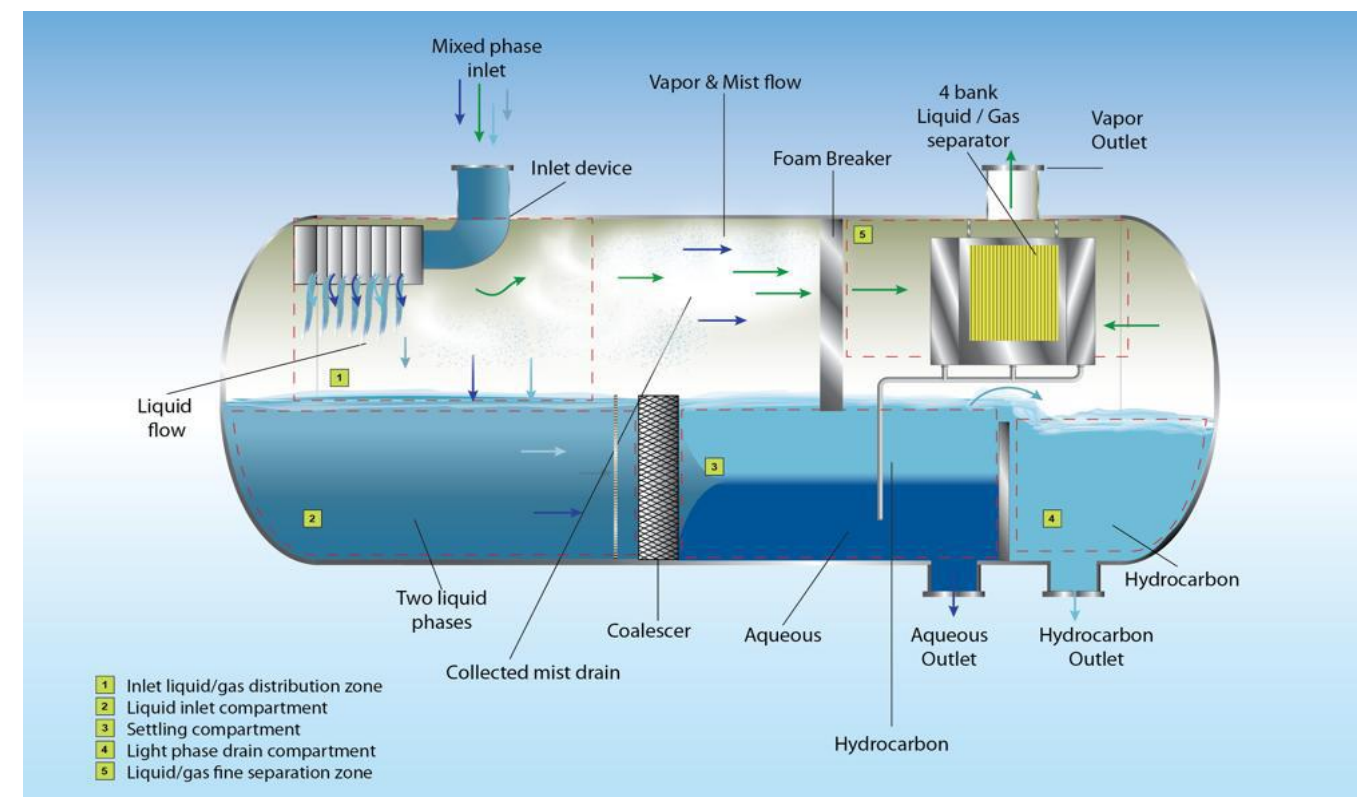

Fig.3. Three-phase gas-oil-water gravimetric separator

The most common separators of this type on the market have a cylindrical tank in which the fluid is introduced from one end through an injection device which can be of different types and shapes, $90^{\circ}$ tubes or others of different efficiency, and which has, immediately after the inlet port, a diverter to redistribute the fluid and create a uniform 
mass flow along the flow direction. In addition to substantially reducing the momentum of the flow, to eliminate turbulence or motions that would nullify the separation, it also carries out a pre-separation operation. They usually consist of plates, vane or T distributors, cones or discs, or tubes with different angles of curvature on which the liquid will impact, losing momentum, unlike gas, which contains less energy, which instead will flow around the diverter towards the separation section.

Once the flow has exited the inlet diverter, it will begin to separate due to gravity and the heavier phase that is the one containing the two liquid components (water and oils), will precipitate towards the area on the bottom of the tank intended for the accumulation of the liquid, on the interface area between liquid and gas. The liquid phase will stay in the liquid collection area (at the bottom of the tank) for the time necessary for the gas bubbles remaining in the liquid to agglomerate through coalescence phenomena and reach the interface area to free themselves in the gaseous phase area. This time is called retention time and in the illustrated devices it is guaranteed by controlling the volume of liquid through floats or weirs. The liquid will then continue its path in the device meeting a coalescing filter in which the oily phase and the water will divide, thanks to the coalescence of the oil drops dispersed in the water, stratifying in the decanting compartment and then be extracted separately at the end of the path. from the bottom of the tank. At the same time, the gaseous phase, after having traveled a stretch in the central area of the tank, in which the droplets retained by the gas not separated by the intake diverter will separate thanks to gravity, will pass through a foam eliminator and then through a two-phase liquid-gas separator to eliminate the last droplets in suspension and exit as a gas from the upper outlet. The design of these devices, in addition to geometric parameters related to the feasibility of installing the device and its operation, must take into account many factors such as:

- The operating temperature. This factor greatly affects the choice of material to build the tank and the various components that will interact with the fluid. Usually expensive metal alloys are used to withstand the high temperatures and strong pressures necessary for the success of the separation process of petroleum fluids. For example, the increase in temperature increases the corrosion of carbon steel by means of $\mathrm{CO} 2$ and $\mathrm{H} 2 \mathrm{~S}$, up to a maximum of $40^{\circ} \mathrm{C}$, beyond which there is a gradual decrease in corrosion due to the high resistance to transport of the corrosive layer with increasing temperature.

- The physical properties of the fluid. The properties of the fluid significantly influence the efficiency of the device and the duration of the process. Fluids with high density difference and low viscosity will require a shorter retention time when compared to less dense fluids.

- Flow rate of the flows of the different phases making up the fluid. To obtain a shorter retention time inside the separator, the injected fluid must have a high inlet speed. This would involve a decrease in the size of the particles dispersed in the substrate, making it difficult to separate the phases, and a considerable increase in the size of the device to avoid the reabsorption of the liquid in the gaseous phase (Elhaj and Adli, 2014).

- Costs (capital and operational). Like all types of industries, even the oil industry tries to obtain the maximum result at the lowest possible cost. For this reason, costs are a fundamental aspect when designing these devices, especially as regards the size, shape and construction materials. 
- Presence of impurities and tendency of the fluid to create foam. The presence of impurities, such as sand or other coarse solid particles, can create serious problems to the separator to avoid which filters and components are often present inside the device to facilitate maintenance and cleaning (Arnold and Stewart, 2008). Furthermore, many hydrocarbon mixtures have a more or less strong tendency to create foam and waxy substances, therefore, chemicals are usually injected into the process in order to prevent these problems that would lengthen the retention time in the separator. Often there are also components called foam eliminators to eliminate the foamy substances formed without the use of external chemical agents.

In the scientific literature, models are used to size gravimetric separators which are mainly based on two theories, the Droplet Settling Theory and the Retention Time Theory.

The Droplet Settling Theory is based on the assumption that a drop of liquid of a certain size will take a finite time to separate from the fluid substrate, traveling a known distance. It is assumed that the particles / droplets have a spherical shape and the entrained liquid tends to fall and separate from the gas flow due to gravity. Each drop accelerates due to its negative buoyancy force until this force is equal to the drag force caused by the injected flow. Gradually the acceleration tends to zero until the drop reaches its terminal velocity. The separation or dragging is determined by this speed. If the velocity is slightly less than the gas flow velocity, the drop will be carried along with the gas. On the other hand, if the velocity slightly exceeds the velocity of the gas flow, the drop will settle and separate from the gas.

The terminal speed can be calculated with the Stoke formula, for low values of the Reynolds number $(<2)$ obtained from the equilibrium of the forces incident on the droplet, weight and buoyancy forces, and considering a drag coefficient $C_{d}=\frac{24}{R e}$, can be calculated by:

$$
V_{t}=\frac{g d_{p}^{2}\left(\rho_{l}-\rho_{g}\right)}{18 \mu_{g}}
$$

For Re> 500 the drag coefficient does not depend on the Reynolds number and results $C_{d}=0,34$ and the Newton's formula is used to express the terminal speed:

$$
\begin{aligned}
& V_{t}=K_{s} \sqrt{\frac{\rho_{l}-\rho_{g}}{\rho_{g}}} \\
& \text { with } \quad K_{S}=\sqrt{\frac{4 g D_{p}}{3 C_{D}}}
\end{aligned}
$$

Mostafaiyan et al., (2014) propose an iterative model based on an evolutionary computational algorithm, focused on the droplet decantation theory and developed with the aim of optimizing three-phase separators to minimize the volume. The choice of optimizing this parameter is aimed at supporting the designer in the case of installations where space is limited. Using a well-defined horizontal three-phase separator as a device analyzed, the most important characteristics for its design were identified on which to base the other parameters. The length, diameter, length of the hydraulic channel, the separation and peak times of the separator have been arranged to meet the goal 
of a project that includes a minimum volume. The project was obtained in an iterative way through the recombination and mutation of the parameters of the previous step, stopping when the set of parameters verified conditions subjected to the computer, such as the condition of ensuring the minimum volume occupied.

The Retention Time Theory, on the other hand, provides a sizing that ensures the retention time in the device is sufficient for the time required for separation. It exploits data from practice or experimental processes to determine the time required for the separation of the mixture into its phases. The calculated time will increase if foaming phenomena are expected or if the density and viscosity of the fluid increase. Although the experiments are easy to carry out, they often provide an overestimation of the retention time, accelerated in reality by coalescence processes, and moreover the design will be the more accurate the greater the number of experiments carried out and the more the conditions in which they were carried out the experiments will be similar to those of the real separator. Arnold and Stewart (1986) provide a model usually used for three-phase separators with a cylindrical tank in which the dimensions of the device are calculated based on the retention time from practice and previous experiments. In this model, both the retention time to eliminate water from the oily phase and to eliminate the latter from the water must be specified

$$
d^{2} L_{e f f}=42000\left(Q_{W}\left(t_{r}\right)_{W}+Q_{o}\left(t_{r}\right)_{o}\right)
$$

\section{Use of CFD simulation}

Often these devices are subjected, after a basic initial design, to a CFD simulation to verify that the flow, during the process, assumes characteristics suitable for separation and to predict its behavior in the face of different arrangements and configurations of the internal components that increase the efficiency of the device, facilitating for example the coalescence phenomena, or modifying the speed of the intake flow.

In the work developed by Lauri Kirveskari, for his Master's thesis at Aalto University (2006), there is a careful analysis of a horizontal two-phase separator, of the configuration shown in Fig.4, in which the speed and efficiency profiles of the device are analyzed as the internal components or boundary conditions change. The software used for the simulation is OpenFoam.

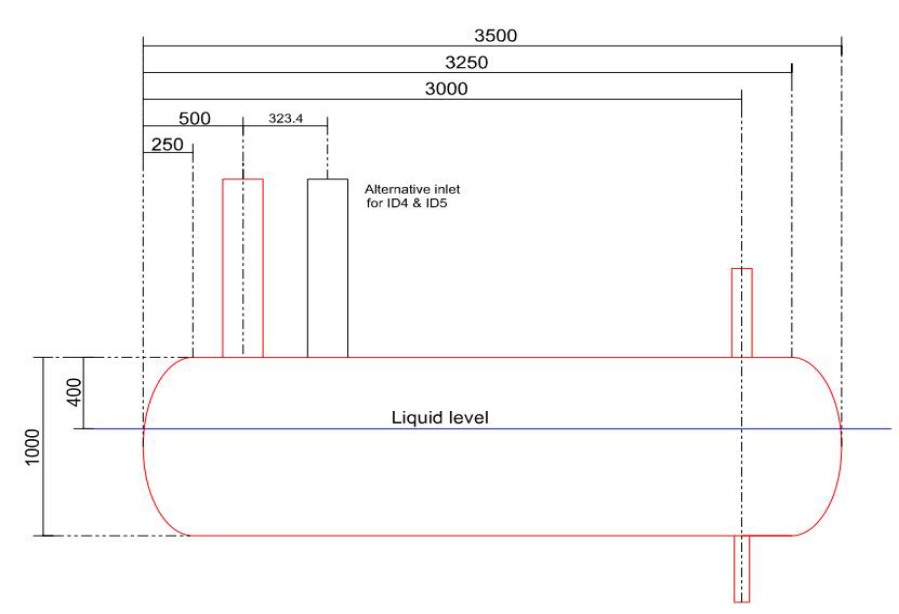

Fig.4. Representation of the separator used for the CFD simulation 
The liquid level inside the tank will significantly change the efficiency of the separator, changing the volume of the part destined for the gas, limiting the reliability of the simulations. The introduced mixture is composed of gas and liquid, considering this last phase as the dispersed one. To measure its efficiency, the yeld of the separator is calculated as the ratio between the quantity of dispersed phase accumulated at its outlet (in this case the liquid) and the quantity of dispersed phase entering:

$$
\eta=\frac{m_{\text {liq,in }}-m_{\text {liq,out }}}{m_{\text {liq,in }}}
$$

From the results obtained from this study, it is clear that an important aspect for the efficiency of the device consists in the choice of the device for introducing the mixture which must ensure a lower entry speed than the phase separation calculated with the Stokes formula. Kirveskari, in his work, studied the variations in efficiency caused by different types of input devices or their absence (Fig.5).

Considering the limitations caused by the existence of various simulation models, both in turbulent (k- $\varepsilon$, Eulerian-Eulerian, $\mathrm{k}-\omega$...) and laminar regime, all involving different errors and reliability of the results, the results obtained by Kirveskari show the higher efficiency of curved inlet pipes (ID3, ID4) compared to plate devices. And in any case, by also simulating the case of the absence of inlet devices, we can see how all the devices introduce greater efficiency compared to the base case (absence of inlet devices), thus demonstrating how the flow velocity profile significantly effects on the efficiency of the separator.

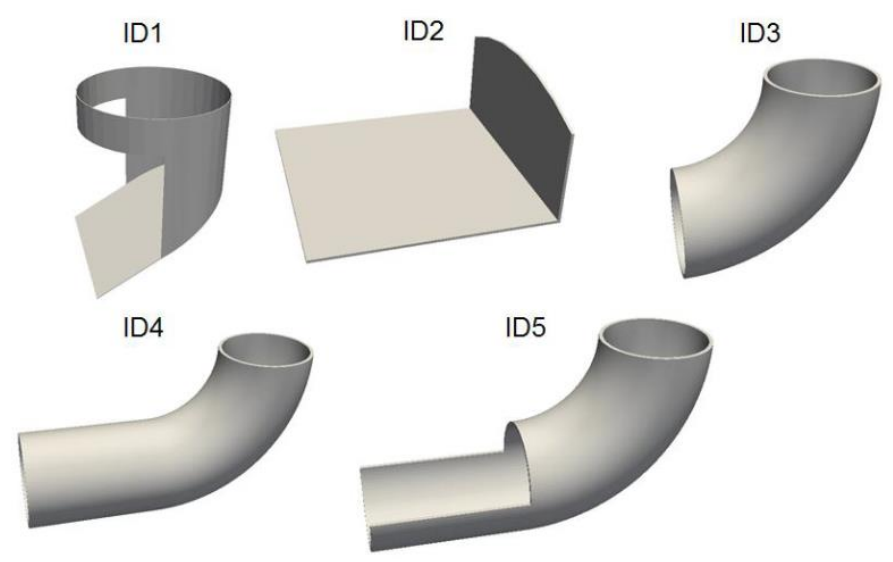

Fig.5. Input Devices

From the graph in Fig.6 it is evident that components ID3 and ID4 are the most efficient, reaching efficiencies above $80 \%$, managing better to limit the speed of entry of the mixture into the device in order to ensure a better operation. The most important result obtained during Kirveskari's analysis shows how the size of the dispersed phase droplets (in this case the liquid) affects even more the internal components of the separator. Kirveskari conducted simulations by increasing the input power of the dispersed phase (bringing it to 200\%, SP200) and increasing the droplet size to 50 microns (compared to 30 microns in the previous simulations, 50_ID4). Placing the results of the quantity of liquid present at the outlet on a graph as a function of time (Fig.7), it is possible to see how the increase in power supply leads to a sudden decrease in efficiency (by increasing the quantity of liquid at the outlet) while how the increase in the size of the droplets leads to an improvement in the efficiency of the separator 
(bringing the quantity of liquid at the outlet almost to zero). These two cases were compared with the basic case of the separator without special entry devices (NoID) and with a droplet size of 30 microns.

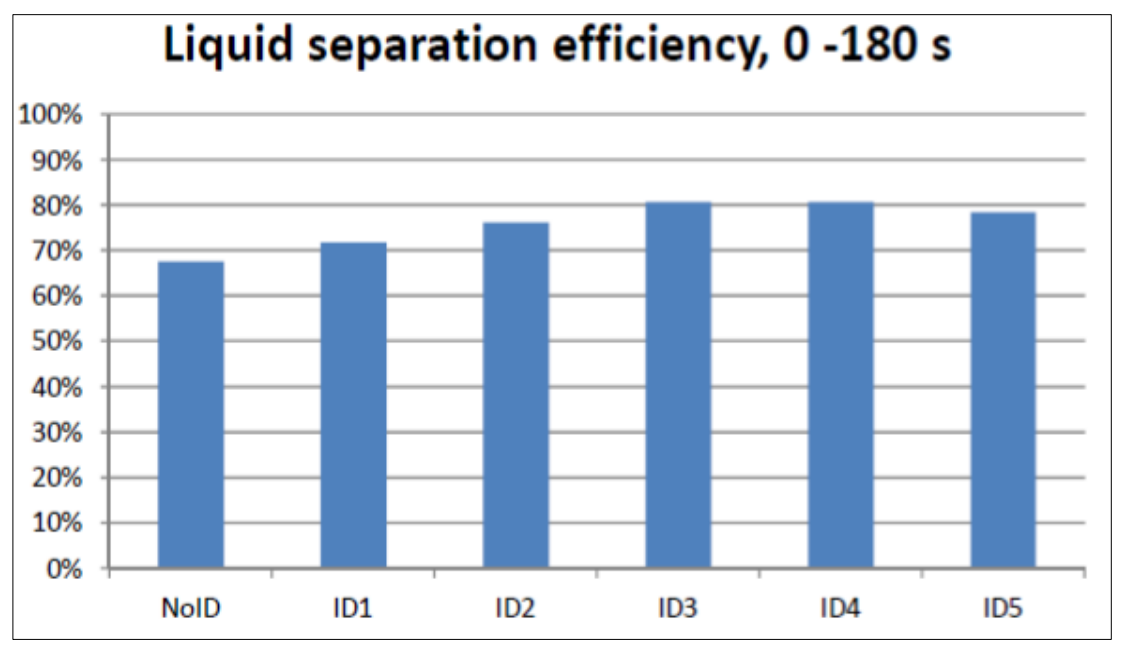

Fig.6. Graph Showing the Separation Efficiency for the Various Input Devices Analyzed

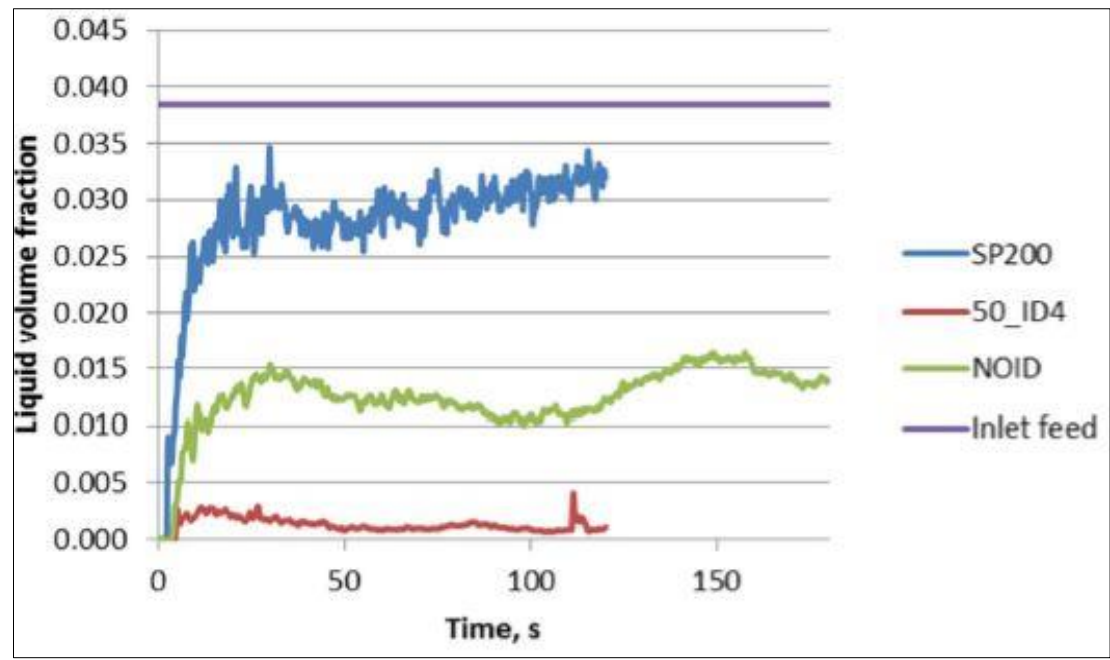

Fig.7. Graph Showing the Fraction of Liquid Volume at the Outlet of the Separator as a Function of Time

It demonstrates how the larger size of the phase droplets dispersed in the mixture facilitates their coalescence and disfavors their breaking, thus making the mixture less stable and therefore easier to separate. The result suggests the presence of a maximum size, below which the droplets are too small to be separated efficiently. There are several mathematical equations used to determine this dimension in the literature. Zhou and Kresta (1997) provide a correlation between the Sauter mean diameter $\left(d_{32}\right)$, which can be measured by observing the mixture and expressing the average diameter of the drops present in the substrate, considered spherical, and the maximum stable diameter $\left(d_{\max }\right)$ through a coefficient $\mathrm{c}$.

$$
d_{32}=c d_{\max }
$$

According to the different types of system, Zhou \& Kresta (1998) declared the value of c oscillating between 0.38 and 0.7. Another approach may be to find a critical Weber number, representing the ratio between the resistant (surface tension) and rupture (surface stress) forces of the drop, at which point the drop is broken (Laleh, 2010). 
$W e_{c r i t}^{\prime}=\frac{\rho \bar{v}^{2} d_{\max }}{\sigma}\left(\frac{\rho_{d}}{\rho_{c}}\right)^{1 / 3}$

With $\quad \bar{v}: \quad$ average speed $\mathrm{m} / \mathrm{s}$

$\sigma: \quad$ surface tension $\mathrm{kg} / \mathrm{s}^{2}$

$\rho_{d}: \quad$ dispersed phase density $\mathrm{kg} / \mathrm{m}^{3}$

$\rho_{c}: \quad$ continuous phase density $\mathrm{kg} / \mathrm{m}^{3}$

According to Laleh (2010), W $e_{\text {crit }}^{\prime}$ it should be around the value 1 for liquid-liquid and liquid-vapor dispersion.

These results show how the efficient operation of these devices is affected by many aspects that are difficult to predict. The best solution would be an experimental study which, however, can require high costs that a simulation with more accurate data such as initial and boundary conditions can reduce, providing the same utility.

The GSMF gravity separator, presented in this paper, is a device that can be used both as two-phase and three-phase separators, in general n-phase, thanks to the presence of appropriate valves and concentration sensors, automatically controlled by devices such as PLC or similar, which can be opened at will and according to the phase to be extracted at the end of the process. The possibility of controlling the valves in a precise way thanks to the presence of sensors, such as conductivity meters that transduce the conductivity of the phase to be extracted in purity, increases the efficiency of the device, making high degrees of separation achievable. The efficiency of the GSMF separator is also guaranteed by the presence of lamellar packs which increase the surface useful for separating the phases by introducing a part of the process in which there are no transverse motions, which would nullify the separation attempt, and which reduce the time of sedimentation needed. It does not have particular structures with inlet deviators present in common separators because the homogeneous and uniform distribution inside the device is guaranteed by the inlet and outlet draining pipes arranged using three-dimensional reverse return techniques that ensure a homogeneous distribution of the loads along the path fluid inside the device. Furthermore, it separates without the aid of mechanical actions or different chemical substances and not constituting the working fluid (solvents) and is made up of components with simple geometries that can be easily found on the market with which to reduce the costs to create and maintain the device.

\section{GSMF Operation and Mathematical Model}

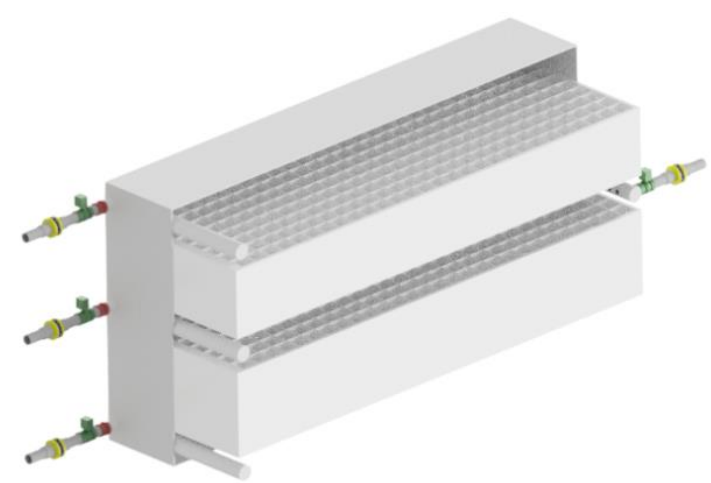

Fig.8. Render of the GSMF Gravimetric Separator 
Through an inlet pressure, supplied by a pump, the fluid entering the device will arrange itself homogeneously in the inlet pipe, consisting of a drainage pipe in PVC or material suitable for the mixture to be separated, closed at one end and with small holes along its length. The flow will be distributed homogeneously in it thanks to the reverse return hydraulic technique, guaranteed by the particular arrangement of the inlet and outlet pipes, so that the pressure drops suffered by each fluid particle are the same because they are led to travel through the same drive in terms of length. Furthermore, always to ensure homogeneous losses along the inlet pipe, and therefore not to create preferential areas for the fluid, the following relationship must be respected between the internal area of the manifold and the sum of the areas of the holes $\left(\mathrm{A}_{\text {foro }}\right)$ :

$$
A_{\text {Dint }} \leq \sum A_{\text {foro }}
$$

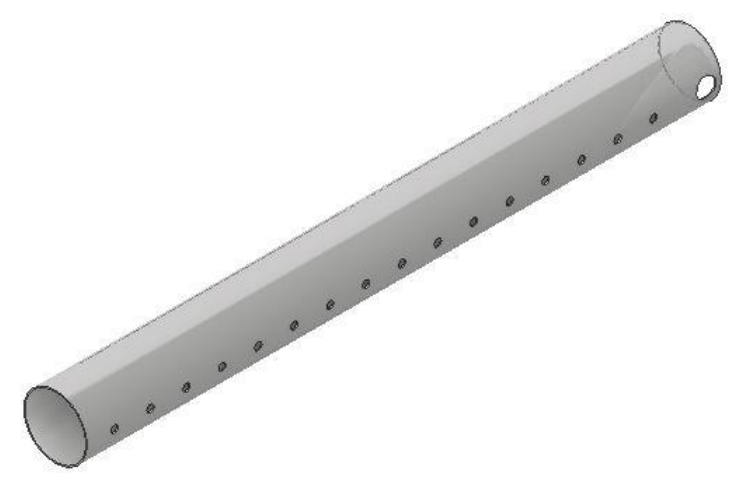

Fig.9. Drainage Pipes used for the introduction, and subsequently the extraction, of the fluid and its phases At this point the fluid will come out of the small holes (Fig.9) forming a laminar flow that will be addressed towards the opposite end, also guaranteed by the reverse return technique for the arrangement of the inlets and outlets. To achieve a laminar motion, the flow must exit the holes with a speed that precisely respects the laminar field.

It can be calculated considering the inlet flow rate $\mathrm{Q}$ and the sum of the areas of the holes, which can be assumed to be equal to the area of the internal diameter of the inlet manifold, making sure that the area of the single hole is such as not to become clogged with pass fluid:

$$
v=\frac{Q}{\sum A_{\text {foro }}}\left[\frac{m}{s}\right]
$$

It can then be checked that it respects the values established by the laminar field by calculating the Reynolds number:

$$
R e=\frac{\rho w d}{\mu}=\frac{w d}{v}
$$

And verifying that it is $R e \leq 1000$.

The flow, being laminar, will allow the different phases present in the mixture to begin to separate due to the action of gravity, as the transverse turbulence is limited. GSFM can be designed so that between the entrance wall and the beginning of the interstices between the honeycombs there is a volume, created by spacing the honeycombs away from the entrance, so that it can be initiated a rough separation of the phases at different densities. Then, once the 
initial separation has taken place, the treated volume will continue its path towards the honeycomb section where the separation will be perfected thanks to the horizontal interstices and the drainage sampling tubes. In the following discussion a three-phase GSMF separator is considered, with two lamellar packs and three horizontal interstices. Entering the section where the honeycomb packs are present, the heavier portions of mixture will hit the walls of the lower shafts, entering them, and the lighter ones in the upper shafts.

Here the real separation will take place, perfecting the separation that occurred previously in any volume present between the entrance and this section of the device. The heavier components will begin a descending vertical motion in which the fluid will not be subjected to horizontal turbulence and will be able to easily overcome the lighter components, which will rise up the shaft to be directed in opposite processes. In the same way, the lighter components will begin an ascending vertical motion in which the fluid, not subjected to horizontal turbulence, can easily overcome the heavier components, which will descend towards the central part to be conveyed in opposite processes. The shafts must have dimensions suitable for the mixture to be separated in order to guarantee the passage of the fluid through them and in a predetermined residence time, so that gravity has the necessary time to act and therefore separate the phases.

Once the sedimentation time has passed, and the time spent in the device, the light components will have reached the upper gap, while the heavy ones will have reached the lower one. The components of medium density, on the other hand, will continue their journey along the central interstice.

At the end of each interstice, once a certain concentration of required substance has been reached, also appropriately measured through concentration sensors such as conductivity meters, the components will come out of their respective drained outlet pipes by means of a specially created vacuum at each outlet. It would be convenient to prepare a second sensor for each outlet positioned at a certain distance from the outlet to ensure that the concentration value measured by the previous sensor is reliable and not dictated by unevenness created by the action of the pump. Therefore, the major part of the separator design work consists in determining the size and shape of the shafts and therefore of the honeycomb packs and the interstices (Fig.10).

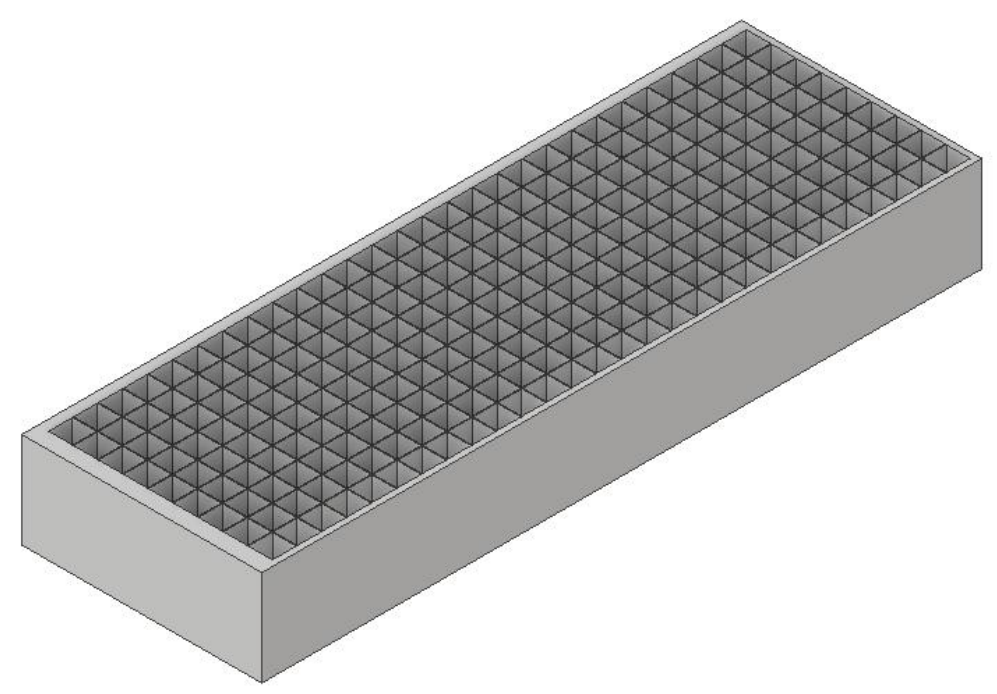

Fig.10. Honeycomb used in the GSMF Separator 


\section{Conclusions}

In this article the main separation techniques of fluid mixtures have been presented and the innovative system called GSMF has been introduced. In a future article, the GSMF device and some of its applications will be described in detail.

\section{Patent}

Lavanga V., Farné S., GSMF https://patentscope.wipo.int/search/en/detail.jsf?docId=WO2016092577 (gravimetric separator mixtures fluid, liquid and gas)

\section{Declarations}

\section{Source of Funding}

This research did not receive any grant from funding agencies in the public, commercial, or not-for-profit sectors.

\section{Competing Interests Statement}

The author declares no competing financial, professional and personal interests.

\section{Consent for publication}

Author declares that he/she consented for the publication of this research work.

\section{Bibliography}

Ahmed, T., Makwashie N., \& Hameed, M., (2017). A review of gravity three -phase separators. J. of Emerging Trends in Engineering and Applied Sciences.

Charles Ross \& Son Company, Mixing Equipment and Applications in the Food Industry.

Baker, J.R., (1991). Motionless mixers stir up new uses. Chem. Eng. Prog., 87: 32-38.

Maurice Stewart e Ken Arnold, Gas Liquid and Liquid-Liquid Separators, (2008).

Batchelor, G.K., The theory of Homogeneous Turbulence, Cambridge University Press, England (1953).

Edward L., Paul, Victor A., Atiemo-Obeng, Suzanne M., Kresta, (2004). Handbook of industrial mixing - Science and practice. CAP. 21-Mechanical Design of Mixing Equipment.

Mostafaiyan, M., Mohammad Reza Saeb, Alireza Emami Alorizi, Maysam Farahani, (2014). Application of evolutionary computational approach in design of horizontal three-phase gravity separators, J. of Pet. Sci. \& Engg.

Elhaj, M. E. M., \& Adli, M. G. A., (2014). Influence of flow characteristics on the design of two-phase horizontal separators. Journal of Science and Technology, 15.

Akram Ghanem, Thierry Lemenand, Dominique Della Valle, Hassan Peerhossaini, (2014). Static mixers: Mechanisms, applications, and characterization methods- A review. Chemical Engineering Research and Design, 92: 205-228. 
Farnè S., Machine. Machine idrauliche. Synopsis, Libreria Universitaria Medea.

www.impianto.it/la-cattura-della-co2.

www.arpae.it - Agenzia regionale prevenzione e ambiente dell'Emilia Romagna.

www.businessinsider.com/in-svizzera-la-prima-centrale-che-cattura-lanidride-carbonica-dallaria-e-la-trasforma-i n-prodotti-utili.

https://www.ingegneriadellambiente.net/ojs/index.php/ida/article/view/261/360. 